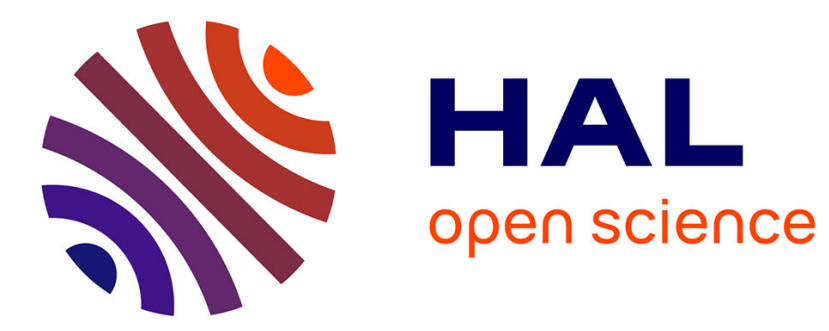

\title{
Dialogues avec la littérature: Louis Chevalier et Jean Duvignaud
}

\author{
Pierre Lassave
}

\section{To cite this version:}

Pierre Lassave. Dialogues avec la littérature: Louis Chevalier et Jean Duvignaud. Genèses. Sciences sociales et histoire, 1999, 34, pp. 114-131. hal-00273873

\section{HAL Id: hal-00273873 \\ https://hal.science/hal-00273873}

Submitted on 16 Apr 2008

HAL is a multi-disciplinary open access archive for the deposit and dissemination of scientific research documents, whether they are published or not. The documents may come from teaching and research institutions in France or abroad, or from public or private research centers.
L'archive ouverte pluridisciplinaire HAL, est destinée au dépôt et à la diffusion de documents scientifiques de niveau recherche, publiés ou non, émanant des établissements d'enseignement et de recherche français ou étrangers, des laboratoires publics ou privés. 


\section{Dialogues}

\section{avec la littérature: Louis Chevalier et Jean Duvignaud}

\author{
Pierre Lassave
}

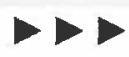

1. D'abord publié en 1958 chez Plon dans la collection "Civilisation d'hier et d'aujourd'hui * dirigée par Philippe Ariès où parurent également Histoire de la folie de Michel Foucault et Baroque et classicisme de Victor L. Tapié, le livre reste encore trouvable aujourd'hui en collection de poche «Hachette-pluriel», 1984.

2. On trouve trace de la relance après-guerre des travaux de démographie urbaine dans les actes de la "Semaine sociologique » du tout jeune Centre d'études sociologiques du CNRS en 1951, in Georges Friedmann (éd.), Villes et campagnes, Paris, Armand Colin, 1953.

3. Voir à cet égard Alain Drouard, «Réflexions sur une chronologie: le développement des sciences sociales en France de 1945 à la fin des années soixante", Revue française de sociologie, vol. $23, \mathrm{n}^{\circ} 1,1982$, pp. 55-85.

4. Louis Chevalier, Les ruines de Subure, Montmartre de 1939 aux années 80, Paris, Laffont, 1985.

5. Jean Duvignaud, L'Oubli ou la Chute des corps, Arles, Actes Sud, 1995.

6. Dont il cite cette éclairante digression méthodologique tirée de Splendeurs et misères des courtisanes:

«Une des obligations auxquelles ne doit jamais manquer l'historien des moeurs, c'est de ne point gâter le vrai par des arrangements en apparence dramatiques, surtout quand le vrai a pris la peine de devenir romanesque. La nature sociale, a Paris surtout, comporte de tels hasards, des enchevêtrements de conjonctures si capricieuses,
$\mathrm{I}$ y a une quarantaine d'années paraissait sous la plume de Louis Chevalier Classes laborieuses et classes dangereuses à Paris pendant la première moitié du XIX ${ }^{e}$ siècle, ouvrage d'histoire sociale devenu aujourd'hui en France un classique de la recherche urbaine ${ }^{1}$. Outre son objet principal - montrer comment le crime se développe et imprime sa marque à la capitale entre la Monarchie de Juillet et le Second Empire - l'essai s'est immédiatement distingué par sa méthode: mêler résolument l'évocation romanesque à l'analyse démographique. Perspective qui a soulevé autant de critiques du côté des spécialistes de la littérature que de celui des historiens, à en croire l'intéressante préface de l'auteur revenant vingt ans après sur son œuvre pour la justifier. S'inscrivant dans le mouvement de renouvellement des études démographiques d'après-guerre, le projet de Chevalier visait à développer sur le modèle d'Ernest Labrousse pour l'histoire économique une histoire sociale fondée sur la «description continue, homogène et chiffrée » du peuplement urbain ${ }^{2}$. Il s'agissait aussi pour lui de détacher l'histoire de Paris des facilités de la citation littéraire impressionniste et incontrôlée. «À quoi bon aller aux documents, et surtout chiffrés, quand il y avait Balzac, Hugo, Alphonse Daudet, Zola et leurs successeurs?» s'exclamait-il par antiphrase à l'encontre des chroniques anecdotiques de l'histoire événementielle. Mais l'ironique préfacier ne laissait pas entendre pour autant qu'il avait cédé à la tentation "quantophrénique» venue d'Amérique et qui, vingt ans auparavant, divisait les sciences humaines entre les tenants de l'explication quantitative des faits sociaux et ceux de leur compréhension qualitative ${ }^{3}$. Sa position était plus subtile: s'il avait vu dans la statistique des migrations, de la mortalité ou de la fécondité un moyen pour libérer l'histoire sociale de sa tutelle littéraire, c'était aussi et en même temps pour étayer, sinon égaler, la qualité cognitive de certains romans. D'un Balzac 


\section{Louis Chevalier: présentation de l'auteur pour Les Ruines de Subure ${ }^{4}$.}

Né à l'Aiguillon-sur-Mer (Vendée) en I9II, Louis Chevalier, ancien élève de la rue d'Ulm, a été professeur $d$ " Histoire et structures sociales de Paris" au Collège de France et professeur d' Histoire du XX siècle»a l'Institut d'Etudes Politiques. La plupart de ses travaux concernent l'histoire de Paris: la formation de la population parisienne au Xax $\mathrm{X}^{\mathrm{e}}$ siècle, classes laborieuses et classes dangereuses à Paris pendant la première moitié du XIX ${ }^{e}$ siècle, les Parisiens, l'assassinat de Paris, Montmartre du plaisir et du crime.

\section{Jean Duvignaud: présentation de l'auteur pour L'Oubli ou la Chute des corps 5 .}

Né à La Rochelle en I92I, Jean Duvignaud, écrivain, sociologue, est l'auteur d'une trentaine d'ouvrages, romans, récits, essais, dont, notamment, Chebika (Gallimard, rg68; Plon/Terre Humaine, r9gI) et le Singe patriote (Actes Sud, rg93). Il est président de la "Maison des cultures du monde» et dirige la revue «Internationale de l'Imaginaire».

surtout, écumeur de rapports savants, lecteur de Malthus, et secrétaire fidèle des «hardiesses du vrai» ${ }^{6}$. En invitant la démographie à valider le récit romanesque, Chevalier ne pouvait manquer de froisser les «gens de lettre», gardiens de la création littéraire, de même qu'en voulant marier la poétique avec la statistique il ne pouvait que provoquer la perplexité des «grands historiens». Sa préface fait preuve en effet d'une certaine ambivalence épistémique: s'il ne veut voir dans le roman social qu'une précieuse source historique à extraire de sa gangue esthétique, il ne semble aussi voir dans l'histoire sociale qu'une source d'inspiration pour un roman qui, mieux que toute étude positive, sait parler du plaisir et du crime, phénomènes majeurs selon lui de la vie urbaine. Il évoque ainsi comme un modèle ce «sociologue quantitatif» de Chicago devenu romancier et avec qui il a visité les quartiers chauds de la ville d'Al Capone. L'évolution des ouvrages de l'historien parisien montrera qu'il a tendu vers ce modèle sans jamais l'atteindre, ni donc s'en prévaloir.

Au cours de cette période, le progrès dans l'exploration scientifique du monde social n'a cependant pas affaibli la dimension cognitive des cuvres littéraires. À l'origine simple source d'informations plus ou moins fiables pour les sciences sociales, le roman est devenu en histoire culturelle un terrain privilégié d'enquête sur les structures imaginaires d'une époque. La sociologie de la littérature en a fait un produit indissociablement esthétique et institutionnel dont les transformations illustrent et activent le changement social ${ }^{7}$. Enfin la «philosophie littéraire» actuelle s'applique moins à saisir la fiction romanesque comme reflet indirect de la réalité ou à l'inverse comme sa déformation imaginaire qu'à relever les performances heuristiques de l'écriture même ${ }^{8}$. Déclin du positivisme aidant, ces dernières exégèses nous rappellent en écho combien les sciences sociales s'interrogent aujourd'hui sur leur propre textualité, du «tournant linguistique» en historiographie au «raisonnement naturel» (ou non poppérien) de la sociologie en passant par la redécouverte de l'anthropologue comme écrivain et non plus simple «écrivant ${ }^{9}$. Nées contre ou tout contre le roman social ou naturaliste, ces disciplines se rendent une à une à leur état de récit. En ces temps vaguement agités par les «Impostures intellectuelles » - voir par exemple l'affaire Sokal - on s'en tiendra ici à l'hypothèse que la tendance postmoderne à la confusion discursive des régimes scientifiques et esthétiques procède de leur distinction 
que l'imagination des inventeurs est à tout moment dépassée. La hardiesse du vrai s'élève à des combinaisons interdites à l'art, tant elles sont invraisemblables ou peu décentes, à moins que l'écrivain ne les adoucisse, ne les émonde, ne les châtre." (Honoré de Balzac, Splendeurs et misère des courtisanes, 1838, Paris, Garnier-Flammarion, 1968, p. 558).

7. On a pu ainsi voir dans le roman du $\mathrm{XIX}^{\mathrm{e}}$ siècle l'expression du héros problématique dans une société bouleversée par le capitalisme libéral et le machinisme, puis avec le capitalisme monopoliste et l'ère des masses du siècle suivant la dissolution du personnage dans le récit et ensuite sa disparition complète de l'énoncé avec le capitalisme réifié en système de signes. Voir entre autres nombreuses références: Michel Zéraffa, Roman et société, Paris, Puf, 1971; Jacques Dubois, L'Institution littéraire, Bruxelles, Nathan-Labor, 1986 ;

Jean-Claude Chamboredon, "Production symbolique et formes sociales: de la sociologie de l'art et de la littérature à la sociologie de la culture », Revue française de sociologie, vol. 27, $\mathrm{n}^{\circ} 3,1986$, pp. 505-529.

8. Grâce à ses digressions pénétrantes, «Proust sociologue » aurait ainsi dépassé en précurseur certains dualismes académiques (macro et micro-sociologie history et story, etc.); transfuge social adoptant les illusions d'optique des cercles qu'il traverse, Marcel révèle, à travers sa passion déçue pour l'ambivalente Albertine, la classe montante des individus composites façonnés par les jeux d'inversion de l'être et du paraître, la dispersion des expériences et des rôles.

9. Sur ces nouvelles perspectives, voir notre étude: ix Retours sur les liens entre sciences sociales et littérature», Cahiers internationaux de sociologie, vol. 104, 1998, pp. 167-183.

10. Voir l'éclairante analyse de Jean-Michel Berthelot d'où il ressort, entre autres conclusions, que l'historien se veut plus proche du vécu quotidien, ne citant que des cas concrets, alors que le sociologue a une perspective plus générale, plus théorisante, davantage orientée vers l'élaboration d'un idéal type: «Histoire et sociologie une affaire de discipline $»$, Recherches sociologiques, $\mathrm{n}^{\circ} 3$, 1998, pp. 23-43.

11. L. Chevalier, Classes laborieuses..., op. cit.

12. On sait qu'il y a deux textes dans Les Misérables: le premier, Les Misères publié en 1848 est le fait de l'académicien enquêteur; le second, repris en 1860 dans l'exil de Guernesey et publié en 1862 , est celui du proscrit et du prophète engagé dans le combat contre toutes les injustices.

13. Victor Hugo, Les Misérables, 1862, Paris, Garnier-Flammarion, 1967, t. II, p. 104. même. D'où notre attention à la nature de leurs relations et interférences que l'on appréhendera à partir de trajectoires précises et comparées d'auteurs diversement situés aux frontières de ces régimes. C'est bien le cas de notre historien ambivalent auquel on adjoindra, à titre de comparaison, l'itinéraire double de Jean Duvignaud, écrivain et sociologue, en respectant une même unité de temps et de lieu. D'autres raisons de ce choix, telle la place respective de ces deux auteurs dans le champ intellectuel, apparaîtront au fil de l'analyse. Mais, bien qu'observant de fait deux cas que l'on pourrait aussi définir par leurs horizons disciplinaires respectifs, l'histoire et la sociologie, notre étude de l'intertexte dans son contexte se gardera cependant ici de toute induction hasardeuse sur leur différences épistémiques ${ }^{10}$.

\section{L'enregistrement des passions}

Bien qu'innovant après-guerre, Classes laborieuses ${ }^{11}$ reste donc un texte sous-tendu par l'opposition classique entre l'imagination romanesque et le fait historique. Chevalier dit s'intéresser d'abord à Balzac, Hugo et Sue parce que l'expertise statistique de la criminalité de l'époque était incapable de saisir les «faits d'opinion» ou de représentations collectives qui influent directement sur les comportements. Il relit ainsi Les Misérables pour y déceler «la contrainte exercée par les faits sur la description», soit moins la description voulue que la description subie par le romancier sous la pression de la rue. Le déplacement de la scène criminelle, de la place de Grève, au cœur de la ville, à la barrière Saint-Jacques, à sa périphérie, devient ainsi le signe spatial de la transformation du peuple dangereux en classe laborieuse. A contrario, les deux versions successives du "Gamin de Paris» donnent un bel exemple du relâchement de la "pression des faits» lorsque l'imagination romanesque reprend le dessus ${ }^{12}$. Ainsi, la 
seconde version désincarne quelque peu Gavroche en oubliant ses tribulations quotidiennes pour l'exhausser en héros de la légende urbaine: «Paris a un enfant et la forêt a un oiseau; l'oiseau s'appelle le moineau; l'enfant s'appelle le gamin. Accouplez ces deux idées qui contiennent, l'une toute la fournaise, l'autre toute l'aurore, choquez ces étincelles, Paris, l'enfance; il en jaillit un petit être. Homuncio, dirait Plaute ${ }^{13}$ ».

Malgré ses professions de foi positiviste, Chevalier accorde peut-être plus qu'il ne le dit à l'imagination romanesque comme le montre, par exemple, la structure même de l'ouvrage. La première partie - «Le thème criminel, son importance et sa signification »est une description multidimensionnelle de la criminalité urbaine; l'auteur y trie ses diverses sources, littéraires et scientifiques, pour bros- ser un tableau cohérent de la misère urbaine. La seconde - «Le crime, expression d'un état pathologique, considéré dans ses causes»reprend le matériau précédent dans une perspective étiologique; l'enchaînement causal de l'exode rural, de l'insalubrité et de la morbidité se présente comme une lointaine application de la méthode des variations concomitantes labellisée par Émile Durkheim. La troisième - «Le crime, expression d'un état pathologique, considéré dans ses effets »enfin et surtout, justifie pleinement le recours aux sources romanesques, seules à même de faire comprendre les drames et les passions de la ville-époque. Dans cette perspective le roman redresse les erreurs de la statistique : la morbidité saisie à l'hôpital reste en effet inférieure à la morbidité réelle car nombre de pauvres refusent d'y aller comme le montrent

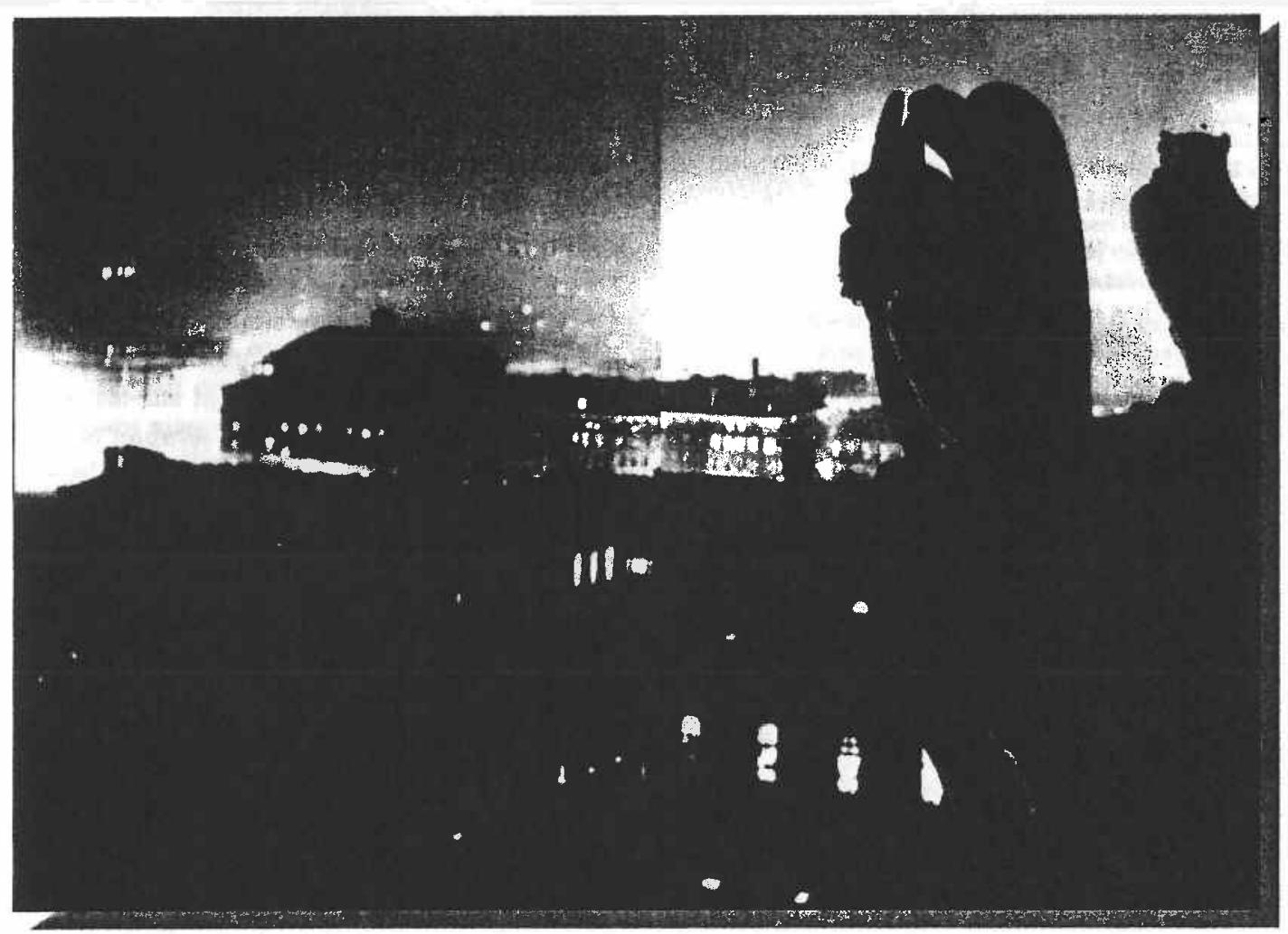

Les chimères contemplent la Cité et Paris, vers 1936. (C) Brassaï 


\section{$\rightarrow$}

14. «Un des grands spectacles où se rencontre le plus d'épouvantement est certes l'aspect général de la population parisienne, peuple horrible à voir, hâve, jaune, tanné. Paris n'est-il pas un vaste champ incessamment remué par une tempête d'intérêts sous laquelle tourbillonne une moisson d'hommes que la mort fauche plus souvent qu'ailleurs et qui renaissent toujours aussi serrés, dont les visages contournés, tordus, rendent par tous les pores de l'esprit, les désirs, les poisons dont sont engrossés leurs cerveaux; non pas des visages, mais bien des masques: masques de faiblesse, masques de force, masques de misère, masques de joie, masques d'hypocrisie ; tous exténués, tous empreints des signes ineffaçables d'une haletante avidité ? Que veulent-ils ? De l'or ou du plaisir?" (H. de Balzac, La Fille aux yeux d'or, 1835), Paris, Garnier-Flammarion, 1988, p. 209.

15. L. Chevalier, Les Parisiens, Paris, Hachette, 1967.

16. L. Chevalier, L'Assassinat de Paris, Paris, Calmann-Lévy, 1977.

17. L. Chevalier, Montmartre du plaisir et du crime, 1980 , Paris, Payot, 1995, p. 35.

18. Ibid., p. 80. les récits de malheur mis en intrigue. De même, le spectacle hallucinant du peuple parisien, «hâve, jaune, tanné», tel que Balzac le peignait dans les premières pages de La Fille aux yeux d'or, exprime dans son emphase l'inquiétude d'une bourgeoisie confrontée à une agglomération humaine qui menace d'exploser ${ }^{14}$. Enfin, le dialogue épistolaire qu'un Eugène Sue entretenait d'un feuilleton à l'autre avec son lectorat populaire - et féminin - pousse l'intrigue et la description des Mystères de Paris vers plus de réalisme - ou moins de zoomorphismes - et de dignité populaire, traduisant par là même le transfert symbolique des classes dangereuses vers les classes laborieuses.

En somme, à l'image de la cire malléable du philosophe empiriste, le roman devient historiquement intéressant pour Chevalier lorsqu'il est façonné par l'enregistrement passif des murmures du monde. Plaintes sourdes ou désirs secrets que le romancier ne peut rencontrer qu'en arpentant la ville en long et en large, en dessus et en dessous. À ces conditions, l'écrivain talentueux fait pénétrer l'historien plus avant dans les raisons des agissements urbains pour y découvrir leur « tréfonds charnel»-que nous traduisons ici en violence sexuelle et criminelle. Telle est la conclusion vaguement sociobiologique que Chevalier tire de son enquête pionnière et qu'il s'est mis à explorer dans plusieurs livres ultérieurs en se rapprochant toujours plus des œuvres littéraires sans jamais toutefois oser franchir à son tour le pas de la fiction.

\section{L'addition fantastique}

C'est d'abord le cas des Parisiens ${ }^{15}$, essai impressionniste sur les types sociaux (fort des Halles, midinette, ouvrier de Belleville, etc.), figures bientôt légendaires de la mémoire urbaine. Tout en les retraçant, l'auteur semble désespérément chercher leur survivance - il 


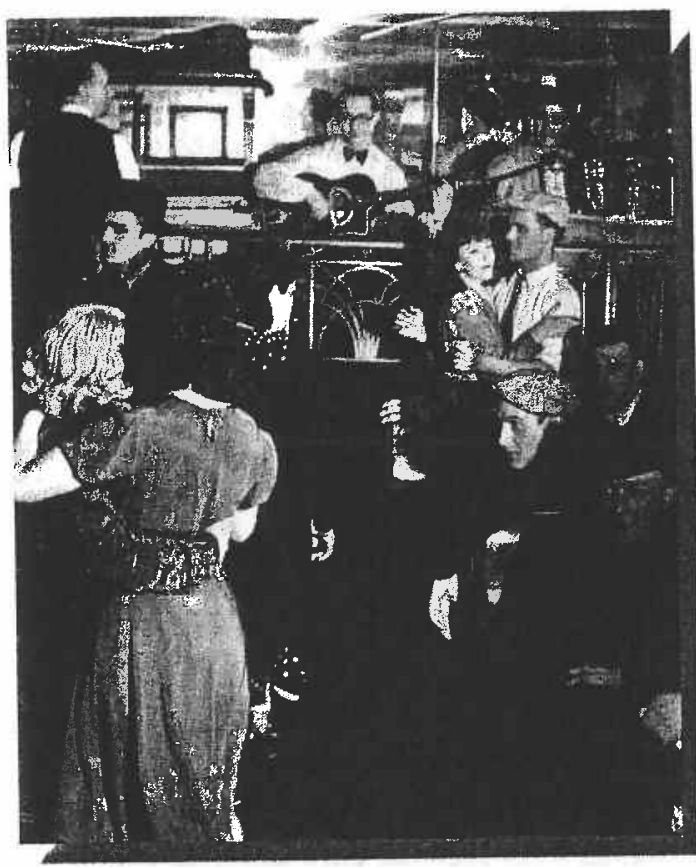

Le bal musette de la Boule rouge, vers $1935-1936$. C Brassai

s'indignera d'ailleurs de les voir détruits dans L'Assassinat de Paris ${ }^{16}$, virulent pamphlet contre l'affairisme et la technocratie qui ont liquidé l'urbanité de la capitale. Mais comme s'il ne voulait pas y croire, l'historien fignole ses figures, de Manon Lescaut à Mimi Pinson en passant par Fantine, et nous parle encore dans ces années soixante d'une certaine Parisienne dont l'esprit accrôtrait le charme du corps et de l'amour lui-même, d'une ville dont le passé libertin imprimerait encore sa marque aux lieux de plaisir, à la différence de Chicago aux relents d'abattoir.

Dans Montmartre du plaisir et du crime ${ }^{17}$, l'hypothèse sociobiologique se précise: «Bien plus qu'à tout le reste à quoi s'attachent innocemment les sociologues du plaisir et $\mathrm{du}$ crime, bien plus que l'intelligence ou l'habileté, c'est la sexualité, et dans ses attributs premiers, qui explique en partie le destin des individus, les activités, les rivalités, les luttes de groupes, de clans, de milieux, c'est-à-dire de gens qui appartiennent à des populations dont les caractères sexuels diffèrent $»$. D'emblée, cette chronique savante du Montmartre de la Commune à la Seconde Guerre mondiale adopte le style narratif libre et à la première personne. Les souvenirs personnels des premières expériences érotiques de l'auteur, les anecdotes au fil de ses rencontres de terrain - cafés, cabarets et hôtels de passe se mêlent aux romans, aux poèmes, aux chansons, aux faits divers. Excepté le rappel de quelques données d'hygiène ou de sécurité publique, les statistiques ne sont plus ici d'un grand usage - «plaisir et statistique ne font pas bon ménage ${ }^{18}$. L'historien brosse d'abord un pénétrant tableau du plaisir parisien avant de retracer son destin montmartrois. La force du hasard révélée par Balzac ou les «jouissances fiévreuses» de la foule des Boulevards célébrée par Baudelaire ont transformé la capitale du siècle dernier en champ magnétique du désir où "les contraires s'attirent». Le chant tragique de la Commune est né à Montmartre, repaire des nouveaux sauvages pour les Versaillais. «L'odeur terrible de la fête populaire » imprégnera longtemps le quartier rebelle à l'ordre bourgeois. Autour de la Grande Guerre, sa réputation de nouveau Subure devient mondiale. Le Tout-Paris, les belles américaines aux bras des escrocs de haut vol font miroiter ses nuits chaudes durant les Années Folles. Le Milieu corse ou marseillais qui tient alors Pigalle avec ses protégés des deux sexes vit en bonne intelligence avec les modestes habitants du quartier: ouvriers, vendeuses, petits commerçants, artistes. Chevalier fait revivre l'envoutant mélange d'ombres et de lumières, de parfums capiteux et de senteurs mouillées de feuilles mortes en choisissant Carco et Mac Orlan. Ce dernier lui apprend «l'addition du fantastique social »: «Un air d'accordéon ajouté à un meurtre donnait au total le mot de l'énigme. Il fallait additionner, additionner tout, le ciel, la lumière des rues, la rouerie des femmes, les 
19. Ibid., p. 223.

20. L. Chevalier, Les ruines de Subure..., op. cit.

21. Pierre Sansot, Poétique de la ville, Paris, Klincksieck, 1984.

22. Notamment sur le quartier de la Goutte d'Or: voir le dossier de la revue Hommes et Migrations, $\mathrm{n}^{\circ} 1122$, 1989. Voir aussi les célèbres romans: Les Allumettes suédoises de Robert Sabatier (1969), La Vie devant soi de Romain Gary/Ajar (1975) et surtout La Goutte d'Or de Michel Tournier (1988). Dans une préface à l'ouvrage collectif La Goutte d'Or, faubourg de Paris (1988), Chevalier s'en tiendra ainsi à Carco pour évoquer les "sortilèges » d'un quartier devenu bazar ouvert au monde.

23. L. Chevalier, Les ruines..., op. cit., pp. 322-324.

24. Ibid., p. 369.

25. Françoise Choay, L'Allégorie du patrimoine, Paris, Seuil, 1992.

26. L. Chevalier, Juanito, Andalousie de boue et de sang, Paris, Stock, 1990.

27. L. Chevalier, Les relais de mer, un village de la côte vendéenne de la veille de la guerre de 14 aux lendemains de la Deuxième Guerre mondiale, Paris, Fayard, 1983. chuchotements du voisin, l'élégance de l'inconnu, le livre que l'on venait de lire et ce que les mauvais garçons de la rue Saint Vincent voulaient bien laisser voir d'euxmêmes ${ }^{19}$ \%. L'historien arpenteur préfère pour cela la révolte d'un Céline contre «l'universelle chiennerie» ou le volcanisme érotique des «promenades obsessionnelles» d'un Miller aux images selon lui surfaites des surréalistes. Car, bien que feignant d'ignorer Freud par prudence disciplinaire, Chevalier ramène toujours le surmoi de la ville à son «ça». Hélas, les derniers bouleversements de son nombril du monde vont le faire déchanter.

\section{Chroniques ethnographiques}

Dans Les ruines de Subure ${ }^{20}$, l'arpenteur revient pour une dernière fois sur son terrain dévasté par les fast-food et envahi par les cars de touristes. Dans l'autobus qui le conduit vers la Butte et ses derniers cafés familiers, il guette à travers les conversations passagères ou sur les façades la moindre bribe ou l'infime étincelle qui ranimerait la mémoire des lieux. Le vieux professeur au Collège de France cède alors à la nostalgie en laissant sa plume s'imprégner de l'argot populaire, à la manière d'un Zola et de tant d'autres maîtres lointainement disparus. Plus sa chronique remonte vers le présent moins le roman ou la statistique lui deviennent utiles. Le souvenir de ses aventures passées de visiteur du soir se mêle aux faits divers d'hier et d'aujourd'hui et à tout ce qu'il voit ou ne voit plus autour de lui. Le style indirect libre de la Poétique de la ville d'un Pierre Sansot (philosophe bachelardien de l'espace urbain) l'inspire ${ }^{21}$. Mais fidèle à sa discipline principale, l'historien ordonne son récit en poursuivant sa chronique de Montmartre à Barbès. D'abord la mise en coupe réglée du quartier sous l'Occupation, «période d'ombre d'autant plus attirante qu'elle était plus épaisse» et durant laquelle le jeune professeur passa quelques nuits chaudes dans les 
hôtels soudainement bondés par le couvre-feu. Après la Libération, Montmartre renoue un temps avec sa légende dorée d'avant-guerre; retrouvailles du plaisir, du Milieu et du monde; les Corses se réinstallent mais les Nord-Africains commencent à disputer la place; les grandes salles de cinéma disparaissent, cabarets et hôtels glissent vers Barbès. Dans les années soixante, l'afflux de migrants du Maghreb et d'Afrique actualise la scène des classes laborieuses et dangereuses. Le bazar et les marchands de sommeil de la Goutte d'Or entament l'image frivole du Montmartre voi$\sin$. De celui-ci, il ne restera vingt ans plus tard qu'un quartier touristique, musée du plaisir disparu, catalogue de boutiques et de restaurants pour agences de voyage.

S'il a montré quelque difficulté à rendre compte des derniers mouvements migratoires qui ont affecté son quartier de prédilection, le chroniqueur s'interdit carrément de nous décrire objectivement ce nouveau Montmartre lessivé. Les enquêtes sociologiques et urbanistiques ne manquent pourtant pas sur le secteur, ni même quelque roman contemporain inspiré par les transformations alentour ${ }^{22}$. Mais, cachant mal son dépit derrière quelques notations chauvines - Paris n'est plus le centre du monde - ou réactionnaires - sur «les crétins soixante-huitards », ou sur «l'absurde centre Beaubourg» ${ }^{23}$ - le chroniqueur noie son chagrin dans quelques derniers cafés où il a encore ses entrées. De là, il préfère nous raconter l'histoire de Luca, un de ses patrons qui assistait blasé du haut de son bar aux ultimes convulsions de Subure mais aussi aux prémices d'une nouvelle aventure urbaine ou tout simplement à l'ordinaire des jours qui passent. Bravant le froid pour assister à l'enterrement de Luca au cimetière Montmartre, le vieil historien communie à nouveau avec le fantastique social en croisant une voyante qui lui affirme que Luca était mort d'avoir trop respiré la poussière des ruines du quartier. "Quia pulvis es... Cette vision d'une histoire dévoreuse de l'histoire, d'un Montmartre venant à bout de Montmartre, ne pouvait que séduire l'historien dont l'humeur, en ce matin d'enterrement, n'était pas à l'allégresse et qui était prêt lui-même à absorber tous les poisons. Mais voilà que la sorcière, passant du constat presque médical à la vision, c'est-à-dire atteignant au sommet de son art, concurrençant les sorciers noirs eux-mêmes, dont on commençait déjà, dans ce petit matin, de distribuer au coin des rues les prospectus étonnants, allait plus loin ${ }^{24}$ ».

Rendu à la frontière du réel et de l'imaginaire, l'observateur de longue durée devenu l'acteur pathétique de l'enterrement de son quartier magique ne franchira pas cependant le seuil de la fiction totale. Les chroniques ethnographiques, genre littéraire qui à travers les histoires de vie et de lieux dédaignés trouve alors ses lettres de noblesse dans «l'allégorie du patrimoine ${ }^{25}$, semblent désormais son lot. En témoigne l'histoire de Juanito $^{26}$, pauvre garçon de ferme en Andalousie qui tente sa chance comme torero. Le professeur d'histoire à rencontré Juanito à Paris par l'intermédiaire d'une de ses étudiantes; Juanito a consigné dans plusieurs cahiers son enfance misérable au village, sa dure vie aux champs, sa tentative ratée pour devenir un célèbre toreador, ses "piropos» charmeurs (déclarations d'amour), etc. Séduit par une authenticité qui ne souffre pas de «vérification tatillonne», l'historien investit tout son talent littéraire pour peindre, à travers l'histoire de Juanito, une «Andalousie de boue et de sang». Après Montmartre puis l'Aiguillon-sur-mer - village natal ranimé par la chronique ethnographique ${ }^{27}$ - Cordoue crée un autre port d'attache d'où entonner un fado nostalgique sur l'humanité menacée par la modernité.

En définitive, dans son cheminement littéraire jusqu'aux portes de la fiction, Chevalier a successivement révélé pour la connaissance 
28. Ajoutons que dans une récente étude des références littéraires mobilisées par les sociologues contemporains, Duvignaud s'avère l'un des plus prolixes à cet égard. Voir Laurence Ellena, «Argumentation sociologique et références littéraires », Cahiers Internationaux de Sociologie, vol. 104, 1998, pp. 33-54.

29. Jean Duvignaud, Le Ça perché, Paris, Stock, 1976.

30. J. Duvignaud, L'Oubli..., op. cit.

31. J. Duvignaud, Le Pandéminium du présent. Idées sages, idées folles, Paris, Plon, 1998. «Dans Le Ça perché, on parlait de soi, dans $L^{\prime}$ Oubli de nous; ici des réponses qu'on fait au Sphinx», est-il écrit au fronton de cet ouvrage.

32. J. Duvignaud, L'Or de la République, Paris, Gallimard, 1957.

33. J. Duvignaud, Pour entrer dans le $X X^{e}$ siècle, Paris, Grasset, 1960.

34. Duvignaud a monté plusieurs pièces de théâtre (le Woyzeck de Büchner, Marée basse de lui-même, etc.) puis écrit sa thèse de sociologie sur le thêâtre précisément, vaste fresque historique sur les «corrélations fonctionnelles» (Gurvitch) entre la scène, l'acteur et l'époque. Son cuvre sociologique se trouve dès lors marquée par l'étude de la stylisation des passions humaines au-delà du langage verbal ainsi que l'ilustre son interprétation du thêâtre antique, shakespearien, classique ou romantique.

35. J. Duvignaud, Chebika, changements dans un village du sud tunisien, Paris, Plon, 1991. de notre époque la qualité empirique du grand roman urbain du $\mathrm{XIX}^{\mathrm{e}}$ siècle (l'enregistrement des passions), la vertu compréhensive des narrateurs contemporains du plaisir et du crime (l'addition fantastique), et, plus prosaïquement, le travail du style pour résister à la perte de la mémoire et du monde (chroniques ethnographiques).

\section{À fronts renversés}

Hybride rare mais significative pour notre objet, l'œuvre de Jean Duvignaud illustre le cas d'un homme de lettres devenu professeur de sociologie de renom tout en continuant à écrire des fictions ${ }^{28}$. À la différence de Chevalier, Duvignaud livre quelques clés de son parcours d'obstacles entre science et littérature grâce à ses essais autobiographiques ou «reconstructions utopiques» ainsi qu'il les définit prudemment; le premier au zénith ${ }^{29}$, le second au crépuscule ${ }^{30}$ auquel il faut adjoindre une sorte de bilan richement illustré de ses idées personnelles et complicités intellectuelles ${ }^{31}$. Tout se passe en effet comme si chacun de ses investissements successifs et alternatifs dans le théâtre, le roman, la critique littéraire, l'essai philosophique et l'enquête ethnographique venait surmonter un obstacle dans l'expérience vécue tout en cherchant à s'affranchir des limites inhérentes à chaque genre.

Très schématiquement, $L^{\prime} O r$ de la République $^{32}$, premier roman édité narrant les aventures sans lendemain d'un jeune réseau libertaire au sein de la Résistance, est présenté après coup comme une manière de se défaire de ses chimères: "s'enfoncer dans la matière de la prose comme Atlan dans le lacis de la couleur» pour se libérer des «idéologies imbéciles, des anxiétés archaïques, le vieil absolu ». Croyances auxquelles auraient un peu trop sacrifié l'ex-khâgneux hegelien qui abandonne son concours sur un coup de tête pour aller à la rencontre de l'Histoire en 


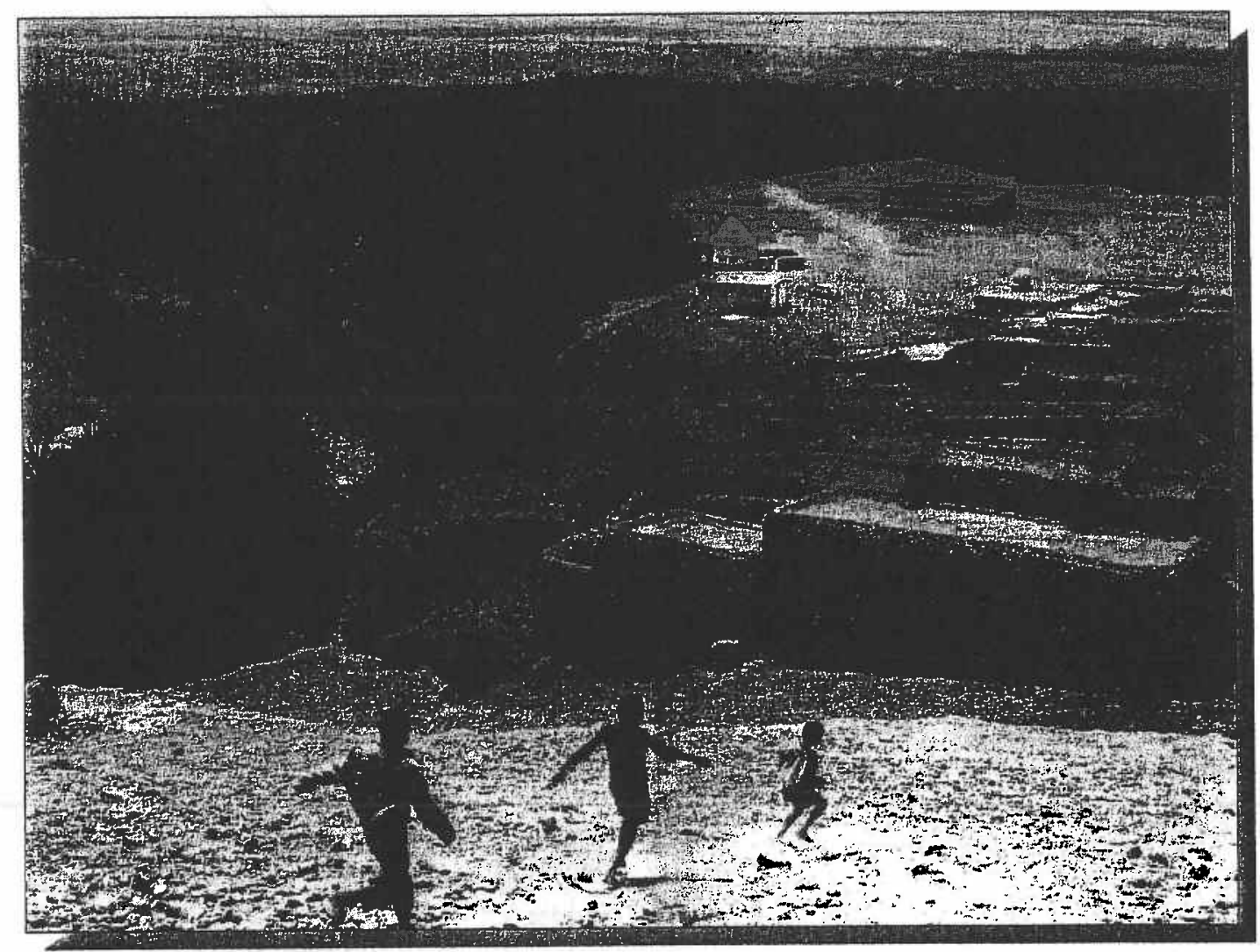

Chebika. (C) Duvignaud

s'engageant dans la Résistance, puis, après la Libération, intellectuel communiste devenu le vicaire libertin d'une improbable révolution. Mais il s'agit aussi, comme le montrent son essai critique Pour entrer dans le $X X^{e}$ siècle (bilan en 1960 de ses contributions à la revue Arguments créée avec Edgar Morin et Kostas Axelos) ${ }^{33}$ et ses incursions marquantes dans le monde du théâtre ${ }^{34}$, de ne pas «réduire la littérature à ses minima", d'écrire l'action des hommes sans laquelle la littérature ne serait qu'une «contemplation morte». Bref, de se placer du côté de la mimèsis plutôt que de celui de la sèmiosis, pour reprendre la distinction classique entre référent et forme. Cet essai, mêlant la réflexion sur «le massacre des idéologies» à la critique du Nouveau Roman - dont le succès d'alors condamne L'Or de la
République - est un appel à «élargir le maigre canton du moi» dans lequel le ghetto littéraire parisien se complairait et à entrer dans le "Royaume des Mères" (Goethe), l'infinité des cultures où «mijote la cuisine du monde».

L'écrivain désabusé - «terroriste défroqué» professeur de philosophie du secondaire devenu l'assistant de Georges Gurvitch, prend à nouveau «le grand large» en allant enseigner la sociologie en Tunisie. Il en revient quelques années après avec ses thèses sur le théâtre et surtout avec Chebika, un texte qui fera date, d'abord paru chez Gallimard en 1968 puis repris et augmenté en 1991 dans la collection ethnographique «Terre humaine» chez $\mathrm{Plon}^{35}$. Ce récit d'enquête sur la vie d'un village de montagne en bordure du désert, 


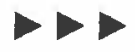

36. Ibid., introd., pp. 13-22.

37. Ibid, p. 429.

38. J. Duvignaud, Fêtes et civilisations, Paris, Weber, 1973 (rééd. Actes Sud, 1991); Le Langage perdu, Paris, Puf, 1973; Hérésie et subversion, essai sur l'anomie, Paris, Anthropos, 1973 (rééd. La Découverte, 1986); L'Empire du milieu, Paris, Gallimard, 1971.

39. J. Duvignaud, Le Favori du désir, Paris, Albin Michel, 1982.

40. J. Duvignaud, La solidarité, liens de sang et liens de raison, Paris, Fayard, 1986; La Genèse des passions dans la vie sociale, Paris, Puf, 1990.

41. J. Duvignaud, Les ombres collectives, sociologie du théâtre, Paris, Puf, 1965; L'acteur, esquisse d'une sociologie du quotidien, Paris, Gallimard, 1965 (rééd. Écriture, 1993); Le Singe patriote, Arles, Actes Sud, 1993.

42. J. Duvignaud, Le Pandémonium..., op. cit.

43. Voir notamment pour ces catégories, Northrop Frye, Anatomy of Criticism, Princeton, Princeton University Press, 1957 (trad. fr. Anatomie de la critique, Paris, Gallimard, 1969) «reconstruction utopique» d'un microcosme aux confins de l'oubli, donne tout son sens au détour par le Sud: «imaginer selon le vrai» en allant au terrain «comme on va à la fontaine» et honorer l'expressivité littéraire en cheminant à travers le labyrinthe des bribes, usages et rites qui mène au sens caché de l'existence commune $^{36}$. «Le village [Chebika] m'a enseigné que la vie sociale, si déçue ou impuissante soit-elle, se détermine toujours au-delà d'ellemême ${ }^{37}$.» En y découvrant le halk, sourd phonème confidentiel qui désigne le mystère de la force créatrice, le chercheur trouve le mot qui unifie ses multiples moi littéraires - romancier, critique, sociologue, ethnologue, etc. - et le situe dans un courant d'anthropologie dynamique - issu de Rousseau, du vieux Fichte, du jeune Marx, de Saint-Simon ou de Proudhon et se manifestant dans les travaux contemporains de Georges Balandier ou de Roger Bastide - alors en butte à la domination structuraliste. En revenant sur nos catégories aristotéliciennes, on dira qu'avec l'anagnôrisis (reconnaissance en forme de révélation), l'auteur dépasse l'opposition entre mimèsis et sèmiosis.

L'imaginaire, le rêve, la fête, le jeu, le rire, le désir, la transe, tout ce que les ordres établis désignent dans l'histoire comme anomique, subversif ou dangereux vont dès lors marquer l'auteur à travers ses expériences vécues ou sollicitées d'homme mûrissant, ses textes de professeur de sociologie de la connaissance, ses romans d'aventures passionnelles et crépusculaires.

Ce thème - dianoïa, faudrait-il alors ajouter à notre panoplie antique - d'un au-delà du discours qui perfore les consciences collectives s'impose comme principe commun de la division du travail entre les genres scientifiques et littéraires auxquels Duvignaud souscrit alternativement. On pourrait ainsi mettre en parallèle les multiples essais sociologiques tels que Fêtes et civilisations, Le Langage perdu ou 
Hérésie et subversion avec L'Empire du milieu $u^{38}$, roman flamboyant du milieu de l'âge où brûlent les passions d'un écrivain - héros miroir de l'auteur - d'une danseuse, d'un acteur, d'hommes et de femmes sillonnant le monde, victimes de leur excessif désir d'absolu. De même, quelques années plus tard, on référerait volontiers Le Favori $d u$ désir ${ }^{39}$ - roman en forme de descente aux enfers de la république de Weimar à la Shoah, allégorie tragique du dédoublement de soi porté à l'hypnose, où Conrad l'esthète désabusé entre dans les rangs nazis pour reconnaître la source du mal et devient le jouet d'agents doubles puis choisit sa mort en un ultime signe de liberté - aux essais sur La solidarité et sur La genèse des passions dans la vie sociale $^{40}$, brillantes dissertations socio-historiques dans la lignée gurvitchienne sur les affinités et les interactions qui échappent aux «champs de servitudes» et se jouent des cadres formels. Dans le même esprit, il faudrait comparer les corrélations fonctionnelles entre les formes de théâtre et les époques développées dans Les ombres collectives et L'acteur avec Le Singe patriote ${ }^{41}$, récit haut en couleur des aventures sentimentales, thêâtrales et politiques de Talma, acteur de théâtre à la charnière de l'Ancien Régime et $\mathrm{du} \mathrm{XIX}^{\mathrm{e}}$ siècle qui atteint la gloire sous l'Empire, haute figure de l'art qui passe à travers les gouttes de sang. Mais on s'en tiendra d'abord ici aux interprétations que l'auteur lui-même, dans ses écrits autobiographiques, suggère des liens ou passages entre ses essais sociologiques et ses œuvres de fiction. À relire ces écrits à la lumière de l'ensemble de sa production, on ne peut en effet qu'être frappé par la récurrence d'images, d'aphorismes, de symboles et de notions ou concepts qui jalonnent le parcours, traversent tous les genres expressifs explorés et constituent de proche en proche un réseau sémantique particulier. S'en dégage l'impression d'une sorte de mythologie personnelle que l'auteur édifie au fil de ses «reconstructions utopiques» et dont le Pandémonium $^{42}$ fournit l'ultime bilan. Voici donc apparaître muthos, autre catégorie de la grammaire aristotélicienne ${ }^{43}$.

\section{Mythologie personnelle}

Notons parmi ces images récurrentes, celle de l'apparition ovationnée du «Petit Monsieur» (Hitler) à l'estrade d'un meeting de rue à Berlin, énergumène aux bottes rutilantes, tragique pantin du grand théâtre de la Modernité; vision à laquelle on peut opposer mentalement celle, non vécue en direct comme la précédente, du «célibataire moustachu» (Nietzsche), grand homme dégingandé au bord de la démence pissant sous l'arcade d'une place de Turin. Émaillés d'un texte à l'autre, les aphorismes répétés condensent la signification des images: «L'homme est le songe d'une ombre» (Pindare) souligne l'éphémère stylisation du monde par le théâtre; «Il découvre la solitude en découvrant la volonté" (Shakespeare) annonce l'avènement de l'individu isolé, roi nu des Temps Modernes, lorsque les prodigues volutes des églises baroques se figent et que s'annonce l'ère comptable des machines du capital; "Les ancêtres redoublent de férocité 》 (Kateb Yacine) rappelle cependant que cette solitude demeure toute relative tant que le vif reste saisi par le mort. Mais dans cette exclamation, «Si le soleil ne brille que pour la bourgeoisie, eh bien camarades, nous éteindrons le soleil!» (Trotsky), un apôtre martyr de la révolution permanente entretient la flamme de l'utopie, celle qui fait dire au savant lucide que "toute société se paie de la fausse monnaie de ses rêves» (Mauss). Reste que «Dieu sauvera les hommes quand ils seront brûlés comme le charbon » (le Coran). Contenant mal son désir d'absolu, l'autobiographe s'assimile aux «terroristes défroqués» et autres «hérétiques» qu'il étudie et met en scène alternativement. Dans son effort de 


\section{$\rightarrow$}

44. «Représentons-nous une grande surface de mur, fraîchement chaulée, au croisement de plusieurs ruelles d'une ville populeuse. Les gens vont et viennent. Bientôt l'on verra, tracée à la craie ou au charbon, une vague figure, à peine esquissée - et qui paraît attendre. Sitôt ce signe apparu, d'autres vont l'accompagner, le prolonger, lui répondre sur la surface blanche. Les passants, au hasard, vont continuer à emplir cette plage vide dont la nudité attire, les uns poursuivant un trait, d'autres continuant une figure commencée, presque aucun ne dessinant une figure achevée. Cet ensemble de figures est-il désordonné ? Quand on y regarde de près, on constate que les passants, sans se voir, sans se connaître, apparemment au hasard, ont fini par délimiter une figure. [...] Les gens de la ville populeuse n'ont fait que compléter une forme qui possédait, par sa structure propre, une force constituante » (J. Duvignaud, Le Ca perché, op. cit., p. 237).

45. Ibid., p. 267.

46. Ibid.

47. Sur le plan littéraire, seul L'Or de la République a été réédité en poche "Folio-Gallimard». Les romans suivants, bien que publiés chez de grands éditeurs (Gallimard, Albin Michel), ont peu marqué la critique. Sur le plan universitaire, l'ex-assistant de Gurvitch, bardé de ses thèses et essais au retour de Tunisie en 1967, a da, à défaut de la Sorbonne ou de l'École pratique des hautes études, postes plus prestigieux, se contenter d'une chaire à l'université de Tours.

48. George Perec, «Tentative d'épuisement d'un lieu parisien», Cause Commune, n 1, 1975; rééd. Paris, Ch. Bourgois, 1975. mise à distance réflexive ( $«$ Regard oblique à travers les choses») le sociologue voit l'univers social se projeter sur un mur de graffiti ${ }^{44}$. Image symbole de la production naturelle et automatique de formes sociales qui traverse tous les récits comme une borne repère.

C'est donc en scrutant le mouvement secret qui crée et recrée ces formes partout et en tous temps que l'anthropologue du halk remonte à la source paradoxale de la créativité humaine. Celle qui impulse le drame de chaque roman, forme communicable de «l'expérience infinie de ce qui pourrait être dans ce qui fut $t^{45} \gg$. D'où il ressort que la fiction n'est plus seulement pour Duvignaud l'exutoire de ses propres aventures sentimentales, idéologiques et professionnelles, mais s'affirme comme manière "d'expérimenter l'ampleur d'une vision du monde possible ou d'en démontrer l'inanité ${ }^{46}$ ». Ces deux fonctions productives de sens se trouvent intimement mêlées dans un parcours qui, au-delà de ses propres rationalisations rétrospectives, transforme ou sublime l'expérience vécue par les deux canaux de la réflexion et de la narration.

\section{Divisions en châne}

La figure de «l'intellectuel total» incarnée par Sartre, tout à la fois philosophe, écrivain et militant, a comme l'on sait longtemps hanté la vie intellectuelle française d'après-guerre. Ce serait probablement réduire le cheminement singulier de Duvignaud entre la fiction et la sociologie que de l'annexer à ce modèle ou à son lointain ancêtre humaniste. Il en irait de même si de surcroît on indexait aux variations de la mode les échappées et passages ici entrevus d'un type d'ouvrage à l'autre. Nul doute que la vogue parisienne du Nouveau Roman n'a pas été étrangère à l'échappée du romancier d'aventures aux arrière-plans autobiographiques et historiques vers les horizons alors plus ouverts de la sociologie puis de 
l'ethnologie. Nul doute aussi que la domination momentanée du paradigme structural sur ces dernières disciplines et la détente sur un front littéraire plus hétéronome ont pu inciter le sociologue de la création culturelle à renouer avec la fiction pour élargir son point de vue. Mais l'écrivain-sociologue, rare de son espèce, ne profitera guère de sa double expérience pour affirmer sa position épistémiquement originale en l'explicitant. De même que dans ses écrits autobiographiques il ne suggère qu'à peine les déboires littéraires et universitaires qui précèdent ses coups de godilles dans ces champs respectifs ${ }^{47}$, de même il n'invoque qu'un vague élargissement phénoménologique de la saisie du monde lorsqu'il met en parallèle la réflexion anthropologique et la fiction romanesque. C'est peut-être dire qu'il intériorise paradoxalement les lois classantes de ces champs en s'intéressant tant à tout ce qui les défie comme l'anomie qu'il étudie, les hérétiques qu'il met en scène et les récits alors atypiques tel celui du Soleil Hopi qu'il célèbre avec Chebika.

La division des genres alternativement pratiqués apparaît alors congruente avec le dédoublement de soi que l'auteur transmet à ses personnages romanesques. Bertrand et Conrad, les héros problématiques respectifs de L'Empire du milieu et du Favori du désir, sont ainsi fatalement partagés entre leur désir de création esthétique et leur volonté d'engagement politique; leurs aventures amoureuses respectives illustrent un tel dédoublement: les femmes qu'ils rencontrent dans la phase d'exaltation esthétique, Nora pour Bertrand, Ottilie pour Conrad, sont blondes, aériennes, apolliniennes; puis celles qui les accompagnent dans leur engagement mortel, Judith et Cecilia, sont brunes, terriennes, dyonisiaques; le jour et la nuit. Tout se passe comme si ces divisions en chaîne trouvaient leur unité ou leur complémentarité en faisant progressivement système: l'ombre de l'imagination ne peut se passer des lumières du raisonnement

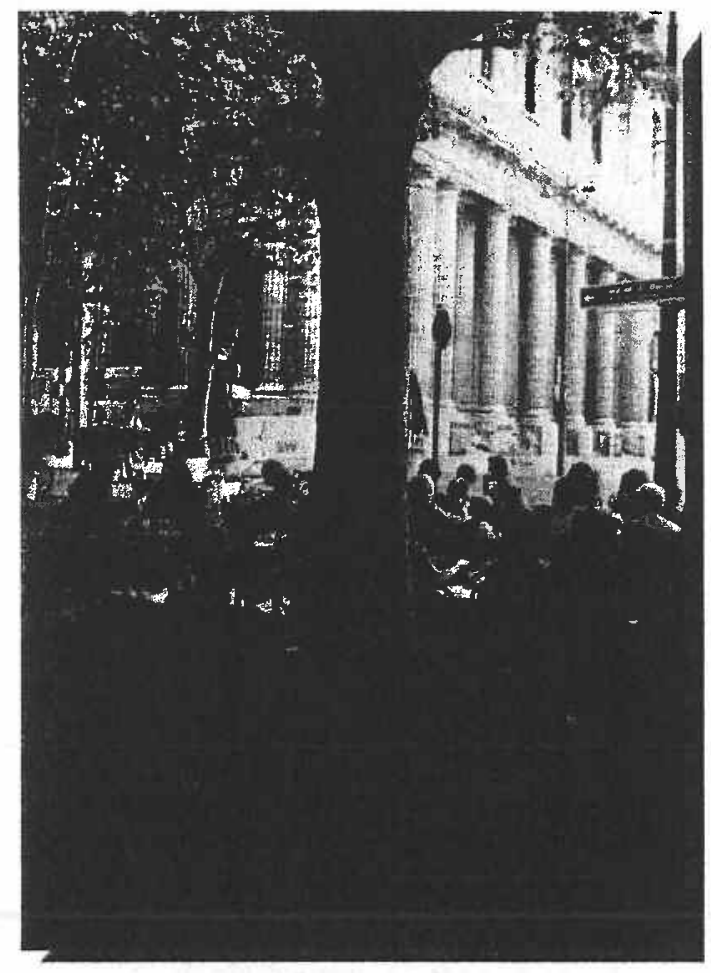

Le Café de la Mairie, place Saint-Sulpice à Paris. Lieu nostalgique où George Perec, élève puis ami de Duvignaud, écrivit "Tentative d'épuisement d'un lieu parisien ${ }^{48}$. (c) Duvignaud

et inversement. L'enfant formé à l'école montessorienne ou l'étudiant passionné de Spinoza affirment donc plus en pratique qu'en théorie qu'il n'y a pas de concept sans sensation, de sociologie de la connaissance sans expérience poétique. La déprise du monde et de ses divisions qui survient au soir de la vie livre peutêtre le fin mot de l'affaire: le dédoublement et l'entre-deux ne sont au fond qu'entrelacement de mots de la même langue, d'un même moi tout à la fois lié et séparé du monde...

«Flâner, rêvasser... Et soudain un déclic, une détente m'éloigne de la vie qui devient un spectacle auquel je suis étranger. Ai-je remisé quelque part mes obligations, mes affaires, les menues rages de la passion? Indifférent comme le serait un dieu caché devant une fourmilière. On est là un niais, un étourdi, on 
49. J. Duvignaud, L'Oubli..., op. cit., p. 202.

50. Dans Les Sociologies contemporaines, Paris, Seuil, 1990, Pierre Ansart définit ainsi quatre grands courants ou écoles en France: le structuralisme génétique (Bourdieu), la sociologie dynamique (Balandier, Touraine), l'approche fonctionnaliste et stratégique (Crozier), l'individualisme méthodologique (Boudon).

51. Dans son Pandémonium Duvignaud livre les pièces d'une de ses rares controverses épistémologiques aux côtés de Gurvitch et à l'encontre du structuralisme naissant à travers l'œuvre de Lévi Strauss qu'il analyse avec déférence malgré l'ironie du titre ( Le vicaire des tropiques», Lettres nouvelles, 1958, repris dans Pandémonium, pp. 115-126). L'anthropologue s'est senti obligé de répondre cordialement et point par point car «sa pensée y était constamment frôlée, mais non saisie».

52. «Il faut affirmer le plaisir du texte contre les indifférences de la science et le puritanisme de l'analyse idéologique; il faut affirmer la jouissance du texte contre l'aplatissement de la littérature à son simple agrément», Roland Barthes, Le Plaisir du texte, Paris, Seuil, 1973, paratexte, quatr. couv.

53. Sur cette célébration, voir Louis Pinto, «Épreuves et prouesses de l'esprit littéraires ", Actes de la recherche en sciences sociales, $\mathrm{n}^{\circ} 123,1998, \mathrm{pp} .45-64$.

54. Dans La nuit des prolétaires, archives du rêve ouvrier (Paris, Fayard, 1981), Jacques Rancière exhume de l'oubli les poèmes, chroniques de l'atelier-prison, lettres d'amour. journaux de revendication des prolétaires du siècle dernier en révolte contre un système qui les condamne à vie à la misère ou au travail manuel.

55. J. Duvignaud, Chebika..., op. cit., p. 37.

56. L. Chevalier, Juanito..., op. cit., p. 17. n'est plus dans le coup. Un état d'attente, et l'on découvre le paysage des autres d'un regard oblique. Au lycée, on me grondait pour ces instants d'absence et, depuis, je me suis souvent écarté ainsi des circonstances, de moi-même. Voilà le seul indice qui me rappelle que je suis le même, à travers les personnages que j'ai habités ${ }^{49}$.»

En somme, Duvignaud a successivement rencontré la double face du roman, d'abord exutoire de l'expérience vécue, puis forme d'exploration imaginaire des possibles dans l'histoire humaine. Mais c'est aussi pour avoir éprouvé la dimension égotiste de cet art, et avoir notamment subi les caprices ou les exclusives de son milieu, que le romancier s'est ouvert à la sociologie qui alors suscitait vocations et offrait une manière de carrière. Mais celle-ci, à peine affiliée dans les manuels de la discipline à la mouvance large de la «sociologie dynamique ${ }^{50}$, s'est déroulée sans provoquer de controverse scientifique ni donc faire "école" ${ }^{51}$. S'ensuit une double trajectoire d'écrivain et de savant dont les propres reconstructions transcendent la division de soi en une sorte d'allégorie, à la fois passionnée et raisonnée, de la subversion des normes ou des genres établis. Déprise professionnelle et hétéronomie disciplinaire aidant, tel parcours tend à terme vers la fusion des codes dans l'essai poético-herméneutique aux arrière-plans autobiographiques.

\section{Aux frontières des genres et des disciplines}

Créateur de fiction à la différence de Chevalier qui prend seulement la littérature classique pour source et modèle, Duvignaud ne rejoint pas moins notre historien dans un commun et tacite «plaisir du texte» 52 . Cet amour partagé et naturalisé des belles-lettres, œuvres de l'esprit consacrées entre toutes, évite peut-être à chacun de se poser la 
Style de qualité pour sites sans qualités, bientôt haut-lieux du récit ethnographique, demain morceaux choisis possibles pour dictées scolaires:

Village de Chebika dans le sud de la Tunisie, aux confins du Sahara...

«Maintenant, le faitte des hautes murailles rosit, prend des tons brique, tandis que passe une volée d'oiseaux gris et jaune au cri perçant, sortes de palombes du désert. Au-dessus de la murette qui surplombe le ravin, brusquement l'horizon prend forme comme chaque matin à cette heure. C'est-à-dire qu'il se développe d'un côté dans le sens du Sahara et, de l'autre, qu'il dessine comme le ferait le lit d'une rivière desséchée, le cours extrême du chott qui, tout a l'heure, blanchira quand le soleil pompera l'eau du sel. Au loin, vers l'Algérie, la terre est phosphorescente, comme si la première lumière du soleil se réfractait sur ces masses de nuit en déplacement vers l'ouest ${ }^{55}$."

Village d'Andalousie où Juanito est né, à une trentaine de kilomètres de Cordoue...

«Un même site escarpé, de refuge, de défense, d'embuscades, qui évoque des siècles d'invasions, de guérillas, de guerres; une brusque surrection de terrain, un relief inattendu que couronnent souvent les ruines d'un château fort du temps des Maures, ou remontant beaucoup plus loin, gigantesque pans de murs ébréchés par les guerres, laminés par les siècles, débris de tours crénelées qui semblent brandir vers le ciel des silhouettes menaçantes ou désespérées. Sur ces hauteurs, entassées à pleins bords, de minuscules maisons blanches qui, lorsqu'elles le peuvent, s'échappent en coulées le long des pentes les moins rudes et se bousculent les unes les autres ${ }^{56}$."

question qui nous intéresse ici des liens cognitifs et tensions institutionnelles entre genres esthétiques et disciplines scientifiques. L'excellence de l'esprit littéraire peut certes faire oublier les antagonismes de style en célébrant unanimement la rencontre authentique de l'intelligence et de l'émotion ou de la connaissance et de l'expérience ${ }^{53}$. Mais nos deux trajets typiques, l'un partant d'une science positive comme la démographie pour atteindre les rivages miroitants du récit ethnographique, l'autre déviant de sa route romanesque pour croiser dans les eaux de l'anthropologie, se complètent finalement pour révéler, à travers leurs schèmes culturels implicites et leurs pratiques d'écriture spécifiques, divers aspects du dialogue aussi intime que tendu entre sciences sociales et littérature. Progressivement et partiellement pour l'un, précocement et entièrement pour l'autre, chacun s'est livré aux charmes équivoques de l'écriture fíctionnelle pour se libérer des carcans idéologiques et méthodologiques. Au prix d'une relative perte de contrôle épistémique, chacun a expérimenté dans cette écriture une manière originale d'exprimer ce que le raisonnement logique laisse informulé: le fantastique social pour l'un, le mystère de la force créatrice pour l'autre. Francs-tireurs de leur discipline, au sens où aucun des deux n'attache véritablement son nom à un mouvement conceptuel ou à une chapelle savante, l'historien et le sociologue se sont ainsi montrés dignes témoins et serviteurs de la suprématie littéraire s'exerçant sur des sciences qui d'ailleurs s'en défendent de moins en moins.

Dans sa quête méthodique des «faits d'opinion en leur tréfonds charnel», difficilement saisissables par la statistique de la violence urbaine, l'historien des classes dangereuses s'en réfère ainsi à Balzac, Hugo et Sue plutôt que d'écumer, par exemple, les écrits obscurs de «la nuit des prolétaires ${ }^{54}$. Dans ses études herméneutiques sur l'anomie, l'hérésie ou la passion, le sociologue fait quant à lui son miel des monuments littéraires - Antigone de Sophocle, Richard III de Shakespeare, Don Quichotte de Cervantès, Le Prince de Hombourg de Kleist, etc. - plutôt que de tenter le diable avec l'enquête de terrain. Mais 


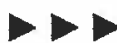

57. J. Duvignaud (et al.), La Banque des rêves, Paris, Payot, 1979 .

58. Sur ces exigences logiques propres aux espaces argumentatifs respectifs de l'histoire et de la sociologie, voir n. 8.

59. Le Linguistic Turn est, rappelons-le, ce mouvement de balancier dans les sciences historiques dont le paradigme, issu notamment de la philosophie analytique américaine, tend à réduire toute pratique ou fait historique au discours qui les constitue. Voir entre autres nombreux commentaires, Geoff Eley, «De l'histoire sociale au "tournant linguistique" dans l'historiographie anglo-américaine des années 1980 ", Genèses, n 7, 1992, pp. 163-193. Dans la même veine contre-positiviste, le «programme fort» de sociologie des sciences réduit tout fait scientifique à sa construction sociale ou au système de croyances et de conflits qui le constitue.

Voir également sur cette abondante littérature:

Dominique Vinck, Sociologie des sciences, Paris, Armand Colin, 1995.

60. On rejoint là peut-être les réflexions historiographiques de Roger Chartier selon lesquelles le discours de l'historien "au bord de la falaise» entre véridicité et littérarité chemine sur un étroit sentier entre la transgression des conventions disciplinaires et le bridage des libertés littéraires: «Histoire, littérature et pratiques », Le Débat, $\mathrm{n}^{\circ} 103,1999$, pp. 162-168. tous deux ne sont pas moins descendus de ces hauteurs pour sacrifier avec bonheur au culte profane des Cultural studies en écrivant l'un Juanito et l'autre Chebika. Études de milieu et récits de vie des déshérités du monde où l'écriture transcende l'objet d'empathie en atteignant à la dimension poétique.

Si l'historien restera probablement l'un des premiers à avoir montré l'apport du roman à la connaissance positive d'une époque ou d'une classe, et le sociologue un des rares à avoir dédoublé sa recherche entre thèse et fiction, l'un et l'autre n'ont pas manqué de faire allégeance à leur discipline et de marquer leur point de vue sur les genres littéraires pratiqués. Les deux professeurs ont ainsi soigneusement évité la psychologie ou la psychanalyse dans leurs enquêtes respectives sur le plaisir et le rêve. L'historien qui tient la sexualité pour loi fondamentale du plaisir et du crime n'évoque nulle part en effet l'économie psychique des liens entre éros et thanatos, ni Sade, Freud ou Bataille. Le sociologue de son côté ne peut certes manquer de saluer dans son «essai d'anthropologie du rêveur contemporain " 57 l'apport clinique du «génial fondateur de la psychanalyse», mais son interprétation sociologiste des discours sur le rêve - correspondances entre thèmes oniriques et milieux sociaux - fait l'impasse sur la dynamique de l'inconscient découverte dans la fameuse Traumdeutung. Enfin, dans ses récits les plus poétiques, l'historien fidèle à Clio a toujours tenu à respecter l'ordre chronologique et le romancier fidèle à ses convictions philosophiques s'est bien interdit de déroger à l'ordre de l'action, des personnages, du sens. On voit ici comment l'habitus disciplinaire en ses contraintes logiques respectives peut modeler le projet esthétique: d'un côté, l'exigence de contextualisation spatio-temporelle ordonne le récit; de l'autre, celle de décrypter le sens du monde structure la fiction ${ }^{58}$. 
Autant donc de limites posées à la confusion des langues, malgré deux œuvres qui mêlent progressivement l'expression imagée ou dramatique du monde à la description curieuse des étrangetés de la vie sociale, passant ainsi d'une sorte de code switching à un code mixing des régimes discursifs, pour reprendre une distinction linguistique connue. C'est dire aussi combien ces deux dialogues singuliers de l'historien et du sociologue avec la littérature révèlent le caractère réducteur de l'assimilation des sciences, aussi humaines soient-elles, à leur dimension discursive ou «littéraire» comme semblent nous y inviter les Linguistic Turn et autre Strong Programme ${ }^{59}$. Mieux vaut en effet éviter les annexions paralysantes si l'on veut explorer les cotes mal taillées ou les jeux situés mais ouverts du contenu et de la forme, des cuvres et des carrières, des styles et des publics. L'analyse circonstanciée de nos deux combinaisons discur- sives confirme finalement notre intuition initiale selon laquelle les interférences croissantes entre régimes scientifique et littéraire se soutiennent paradoxalement de leurs différences principielles. Cette étude de cas, aussi exploratoire soit-elle, montre en effet comment deux chemins allant de la séparation ou $\mathrm{du}$ dédoublement entre les disciplines et les genres vers leur hybridation relative se distinguent par les limites qu'ils imposent précisément à la confusion totale des codes ${ }^{60}$. Où l'on voit donc les traditionnels clivages entre le discours du vrai et du beau refaire surface au moment même où l'on entrevoit leurs liaisons plus ou moins secrètes ou dangereuses. Retour à la surface comme à la réalité, sinon à l'évidence: l'intertextualité tient aux pactes d'écriture ou de lecture respectifs et contingents qu'elle articule. Jeu subtil de rapprochement et d'éloignement des styles que l'on a tenté ici de repérer dans ses nuances singulières. 